\title{
Abondance du potamochère, Potamochoerus larvatus, dans les savanes du Nord-Ouest de Madagascar et risques épidémiologiques associés
}

Antoine RoulLLÉ1, 2, 3

Miguel PEDRONO ${ }^{1,2}$

Éric RAKOTOMALALA ${ }^{2}$

Vladimir GROSBOIS ${ }^{1}$

Ranto RAMY-RATIARISON 2, 4

François ROGER ${ }^{1}$

${ }^{1}$ Cirad

Upr Agirs

34398 Montpellier Cedex 5

France

${ }^{2}$ Cirad

Upr Agirs

BP 853

Antananarivo 101

Madagascar

${ }^{3}$ Université de Rennes I

Campus de Beaulieu

CS 74205

35042 Rennes Cedex

France

4 Université d'Antananarivo Faculté de médecine vétérinaire Antananarivo 101

Madagascar

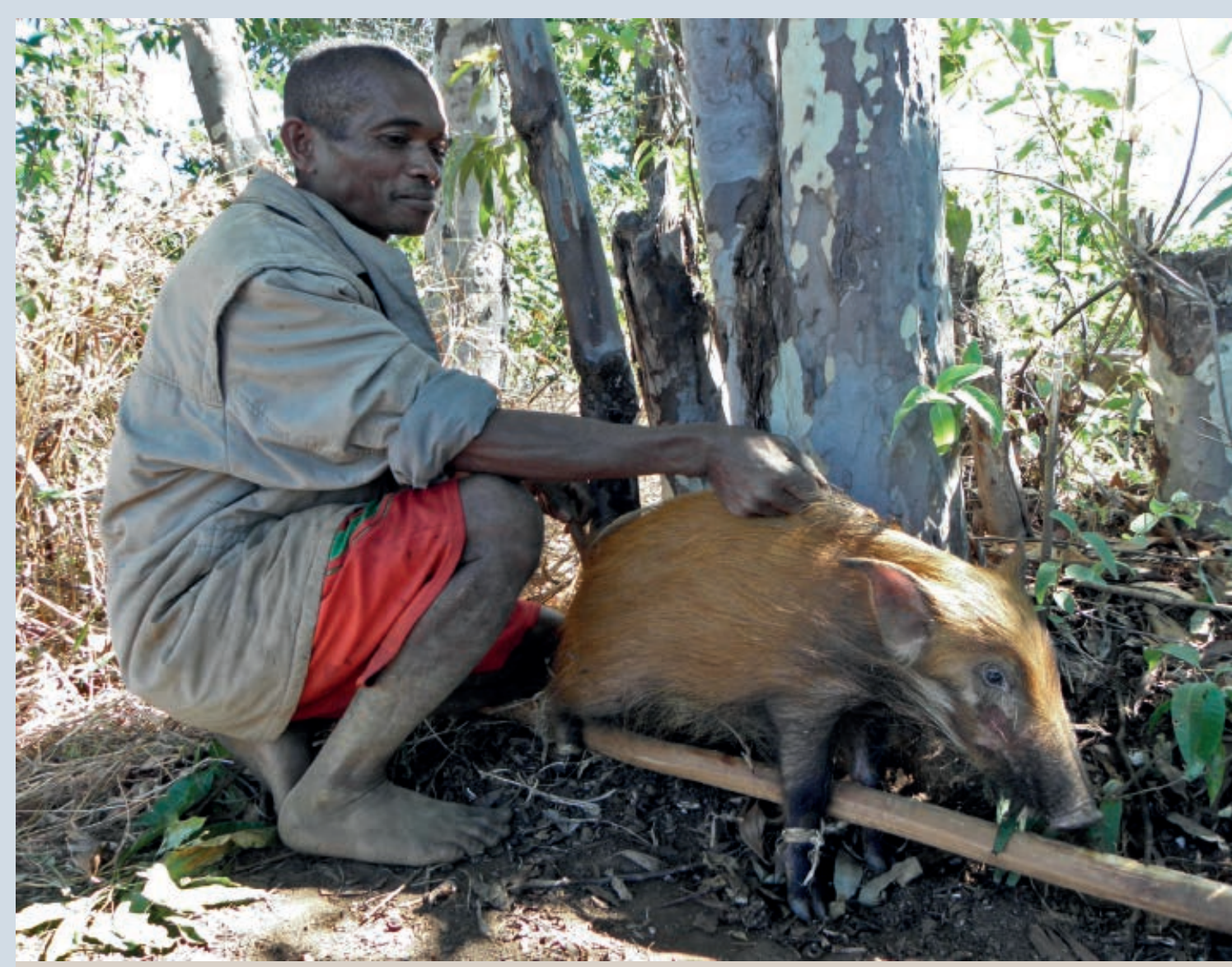

Photo 1.

Jeune potamochère (Potamochoerus larvatus) capturé en périphérie du Parc national de l'Ankarafantsika pour être consommé.

Photo M. Pedrono. 


\section{RÉSUMÉ}

\section{ABONDANCE DU POTAMOCHÈRE, POTAMOCHOERUS LARVATUS, DANS LES SAVANES DU NORD-OUEST DE MADAGASCAR ET RISQUES ÉPIDÉMIOLOGIQUES ASSOCIÉS}

Le potamochère, Potamochoerus larvatus, est un réservoir sauvage potentiel de maladies enzootiques à Madagascar. Parmi celles-ci, la peste porcine africaine (Ppa) est une des plus inquiétantes. Diagnostiquée dans ce pays depuis 1998 , cette maladie virale majeure d'un point de vue socio-économique est inféodée aux suidés et y est encore aujourd'hui très répandue. La caractérisation des voies de transmission du virus entre potamochères et porcs domestiques est une étape préliminaire indispensable à la mise en œuvre d'une prophylaxie sanitaire contre la Ppa. Cette étude a pour objectif de préciser le risque de transmission de la maladie entre potamochères et porcs domestiques dans les savanes périphériques du Parc national de l'Ankarafantsika, dans le Nord-Ouest de Madagascar, où sont présents de nombreux élevages porcins. La méthode des Indices kilométriques d'abondance a permis de mettre en évidence une augmentation significative de l'abondance de ces suidés sauvages hors de la forêt durant la période de fructification de Strychnos spinosa. Le risque de transmission de la Ppa durant cette période apparaît donc plus élevé dans les sites où sont présents ces arbustes. Sur le plan épidémiologique, la maturité et l'occupation spatiale de S. spinosa peuvent servir d'indicateurs de présence du potamochère dans l'ouest de Madagascar. Son utilisation pour adapter les pratiques d'élevage dans une optique de réduction des contacts pourrait limiter le risque de contamination potentielle par le virus de la Ppa ou d'autres agents pathogènes transmis par les suidés sauvages.

Mots-clés : Strychnos spinosa, peste porcine africaine, Potamochoerus larvatus, écologie, abondance, risque épidémiologique, Madagascar.

\section{ABSTRACT}

\section{RESUMEN}

\section{ABUNDANCE OF THE POTAMOCHOERUS LARVATUS BUSH-PIG IN THE SAVANNAH ZONES OF NORTH-WESTERN MADAGASCAR AND ASSOCIATED EPIDEMIOLOGICAL RISKS}

The Potamochoerus larvatus bush-pig is a potential reservoir of enzootic diseases in the wild in Madagascar. Among these, African swine fever (ASF) is one of the most alarming. Diagnosed in the country in 1998, this specific viral swine disease is still widespread. Characterising the transmission of the virus between domestic pigs and wild pigs is an essential preliminary step before implementing health measures against ASF. This paper reports on a study to determine the risk of disease transmission between wild pigs and domestic pigs in the savannah zones of Ankarafantsika National Park in north-western Madagascar, where there are many rural pig farms. The Kilometric Abundance Index method used showed a significant increase in the abundance of wild pigs outside forests during the fruiting period of Strychnos spinosa. The ASF transmission risk in the savannah zone during this period is therefore higher in sites where these shrubs occur. Epidemiologically, the mature fruit can serve as an indicator of bush-pig presence. Using this indicator to adapt farming practices with a view to reducing contact could limit the potential risk of contamination by the ASF virus or other pathogens carried by bush-pigs.

Keywords: Strychnos spinosa, African swine fever, Potamochoerus larvatus, ecology, abundance, epidemiological risk, Madagascar.

\section{ABUNDANCIA DEL POTAMOQUERO, POTAMOCHOERUS LARVATUS, EN LAS SABANAS DEL NOROESTE DE MADAGASCAR Y RIESGOS EPIDEMIOLÓGICOS ASOCIADOS}

El potamoquero, Potamochoerus larvatus, es un potencial reservorio silvestre de enfermedades enzoóticas en Madagascar. Dentro de estas enfermedades, la peste porcina africana (PPA) es una de las más alarmantes. Diagnosticada en este país desde 1998, esta enfermedad viral propia de los suidos tiene importantes repercusiones socioeconómicas y sigue estando muy extendida. La caracterización de las vías de transmisión del virus entre potamoqueros y cerdos domésticos es una etapa preliminar indispensable para el establecimiento de medidas de profilaxis sanitaria contra la PPA. Este estudio tiene como objetivo determinar el riesgo de transmisión de la enfermedad entre los potamoqueros y cerdos domésticos en las sabanas periféricas del Parque Nacional de Ankarafantsika, en el noroeste de Madagascar, en donde abunda la cría porcina. El método de los Índices Kilométricos de Abundancia permitió evidenciar un aumento significativo de la abundancia de estos suidos silvestres fuera del bosque durante el período de fructificación de Strychnos spinosa. El riesgo de transmisión de la PPA durante este período es, por tanto, mayor en los lugares en donde crecen estos arbustos. Desde el punto de vista epidemiológico, la maduración del fruto y la ocupación espacial de S. spinosa pueden servir como indicadores de presencia del potamoquero en el oeste de Madagascar. Su utilización para adaptar las prácticas de cría con el fin de reducir los contactos podría limitar el riesgo de contaminación potencial por el virus de la PPA u otros agentes patógenos transmitidos por los suidos silvestres.

Palabras clave: Strychnos spinosa, peste porcina africana, Potamochoerus larvatus, ecología, abundancia, riesgo epidemiológico, Madagascar. 


\section{Introduction}

Les suidés sauvages sont des réservoirs connus pour un grand nombre de virus, bactéries et parasites transmissibles aux porcs domestiques et pour certains aux humains : peste porcine classique, brucellose, hépatite $E$, tuberculose, leptospirose, trichinellose, etc. (MENG et al., 2009). Parmi celles-ci, la peste porcine africaine (Ppa) est l'une des plus sévères maladies transfrontalières des porcins (COSTARD et al., 2009a). Ses conséquences socio-économiques peuvent être majeures (ALONSO, 2013).

Depuis son diagnostic à Madagascar en 1998 (GONZAGUE et al., 2001), suite à une introduction probable depuis le Mozambique en 1997 (ROGER et al., 2000 ; ROUSSET et al., 2001), la Ppa a sévèrement affecté la production porcine nationale (GONZAGUE et al., 2001 ; ROGER et al., 2001), et persiste en tant que maladie enzootique, maladie présente chez l'animal, sur l'ensemble du territoire (COSTARD et al., 2009b). La Ppa est une maladie spécifique des suidés due à un virus de la famille des asfarviridés très contagieux et pour lequel il n'existe aucun vaccin efficace (PENRITH et al., 2004). Les connaissances générales relatives à son épidémiologie et à son cycle sylvatique/selvatique $^{1}$ avaient permis de suspecter l'intervention de deux acteurs : un vecteur biologique, la tique du complexe Ornithodoros moubata dont la présence à Madagascar a été démontrée (ROGER et al., 2001) ainsi que son infection par le virus de la Ppa (RAVAOMANANA et al., 2010), et un réservoir naturel potentiel, le potamochère Potamochoerus larvatus (ROGER et al., 2001 ; JORI, BASTOS, 2009).

Toutefois, les rôles potentiels du potamochère dans l'écologie du virus de la Ppa et du cycle de la maladie restent à déterminer (RAVAOMANANA et al., 2011 ; JORI et al., 2013). D’une part, le manque de données épidémiologiques dû au faible nombre d'échantillons analysés à ce jour (RAVAOMANANA et al., 2011) et, d'autre part, le manque de connaissances sur l'écologie du potamochère à Madagascar limitent les efforts en cours pour y parvenir. Les seules études réalisées à ce jour sur son écologie l'ont été dans les forêts humides du Nord-Est (ANDRIANJAKARIVELO, 1999, 2000).

La mise en œuvre d'une prophylaxie sanitaire relative à la gestion de la maladie (surveillance, contrôle) nécessite, dans un premier temps, l'identification des habitats pour lesquels existe un risque de transmission du virus de la Ppa. Il se transmet par contact direct ou indirect (HUNTER et al., 2006 ; COSTARD et al., 2013). Un risque de transmission entre potamochères et porcs domestiques existe - dans les deux sens - dans les zones où les deux espèces de suidés partagent le même espace. La divagation des porcs dans les élevages ruraux malgaches les expose à un risque de contamination par la Ppa et expose les potamochères à une infection. Dans les faits, c'est lorsque les porcs domestiques circulent aux abords des villages qu'ils pénètrent potentiellement sur des espaces fréquentés par les potamochères. En majorité, les villages sont ceinturés de savanes arbustives qui constituent l'un des habitats de prédilection des potamochères.

${ }^{1}$ Les termes « sylvatique » et « selvatique » sont ici utilisés respectivement avec le sens de forestier et de sauvage, relatif à la forêt naturelle tropicale.

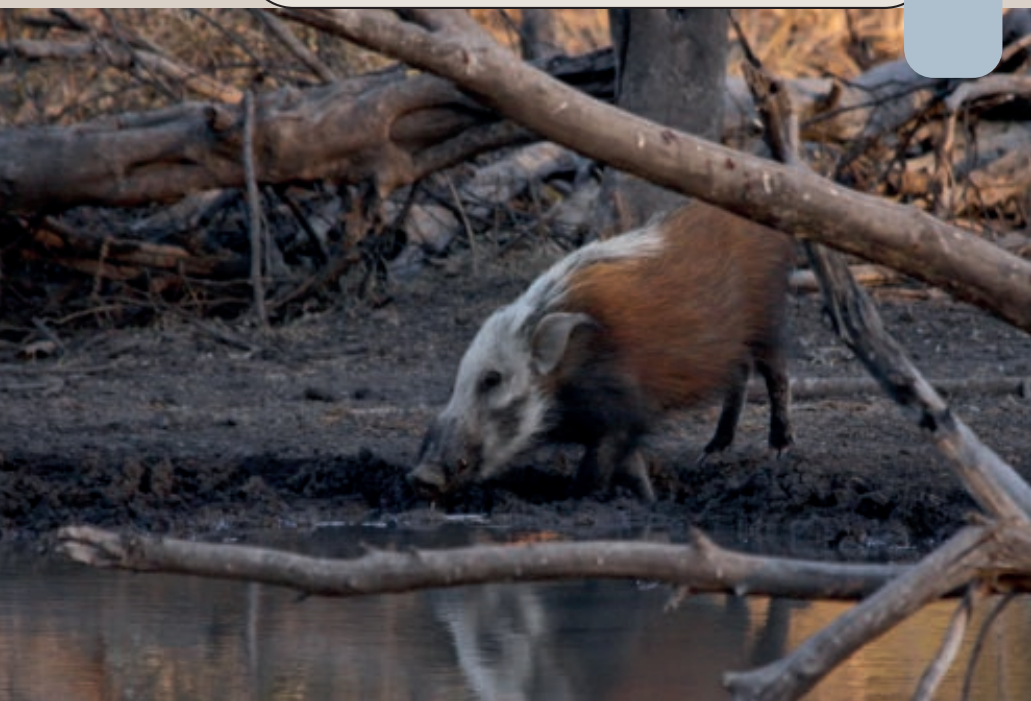

Photo 2.

Potamochère adulte, Potamochoerus larvatus, dans son habitat.

Photo D. Keats.

Potamocherus larvatus est la seule espèce d'Artiodactyle présente à Madagascar, et le plus grand vertébré terrestre sauvage depuis l'extinction de la mégafaune malgache il y a 1400 à 500 ans (BURNEY et al., 2004) (photos 1 et 2). Cette espèce aurait été introduite par l'homme à Madagascar depuis le continent africain, il y a moins de 2300 ans (AMILLS et al., 2013). En fonction de son origine géographique, de sa période d'introduction, et en corollaire de son exposition préalable au virus de la Ppa en Afrique, le potamochère malgache peut être ou non réceptif ou sensible au virus de la Ppa.

Le potamochère possède une capacité d'adaptation importante lui permettant d'exploiter des habitats et zones climatiques très variés à Madagascar (forêt tropicale humide, forêt tropicale sèche, savane boisée, maquis xérophytique). Toutefois, sa présence est conditionnée par la disponibilité en nourriture et en eau, mais également par l'existence d'un couvert forestier ; les milieux essentiellement dominés par des graminées ne permettent pas son maintien (ANDRIANJAKARIVELO, 2003 ; GARBUTT, 2007). Il a un régime omnivore (graines, fruits, tubercules, rhizomes, et petits animaux) variant de façon importante selon les disponibilités alimentaires et la saison. La taille de son domaine vital en Afrique du Sud se situe autour de $7 \mathrm{~km}^{2}$, avec d'importantes fluctuations saisonnières (SEYDACK, 1990). Ce suidé vit en groupes familiaux de 2 à 10 individus composés d'une femelle et d'un mâle dominant, seuls reproducteurs, accompagnés de une à deux générations de descendants (SEYDACK, 1990). Par ailleurs, il représente un gibier très convoité pour les villageois malgaches (GARCIA, GOODMAN, 2003).

Cette étude vise à caractériser les facteurs écologiques déterminant l'abondance des potamochères dans les savanes périphériques du Parc national de l'Ankarafantsika dans le Nord-Ouest de Madagascar. Il s'agit à terme de fournir des données pour estimer les risques de transmission du virus de la Ppa ou d'autres agents pathogènes, et ainsi de proposer des recommandations adaptées en termes de gestion de la Ppa et d'autres maladies importantes pour l'élevage porcin ou à caractère zoonotique, transmissibles de l'animal à l'homme. 


\section{Méthodologie}

\section{Site d'étude}

Le Parc national de l'Ankarafantsika, au Nord-Ouest de Madagascar (figure 1), abrite une population importante de potamochères. Ce plateau sableux de $1300 \mathrm{~km}^{2}$ est majoritairement recouvert de forêt tropicale sèche semi-caducifoliée, le plus grand massif du pays. Le climat y est caractérisé par une saison humide et chaude de novembre à avril, et par une saison sèche et plus fraîche de mai à octobre. La savane arbustive, second habitat du parc, correspond principalement aux espaces périphériques du massif forestier. Généralement milieu de transition entre la forêt et les milieux plus anthropisés, il s'agit d'une formation herbeuse dominée par Aristida sp. et Heteropogon sp. associées à Hyparrhenia sp. et Panicum sp. Des espèces arbustives y sont présentes à l'exemple de Latania lontaroides et de Strychnos spinosa, un arbuste fruitier de la famille des Loganiaceae dont la période de fructification s'étale de la mi-avril à la mi-août (ANGAP, 2007).

\section{Choix de la méthode d'étude des populations : l'Ika (indice kilométique d'abondance)}

Le comportement craintif et nocturne du potamochère (GARBUTT, 2007) limite les possibilités d'observations visuelles directes. Dès lors, les méthodes de comptage indirectes sont préférables aux méthodes de comptage directes. Celles-ci permettent une estimation des populations d'animaux sauvages sur des zones ouvertes très étendues sans recourir à d'importants moyens (MARQUES et al., 2001). L'étude réalisée étant destinée à identifier les facteurs écologiques influençant la présence des potamochères,

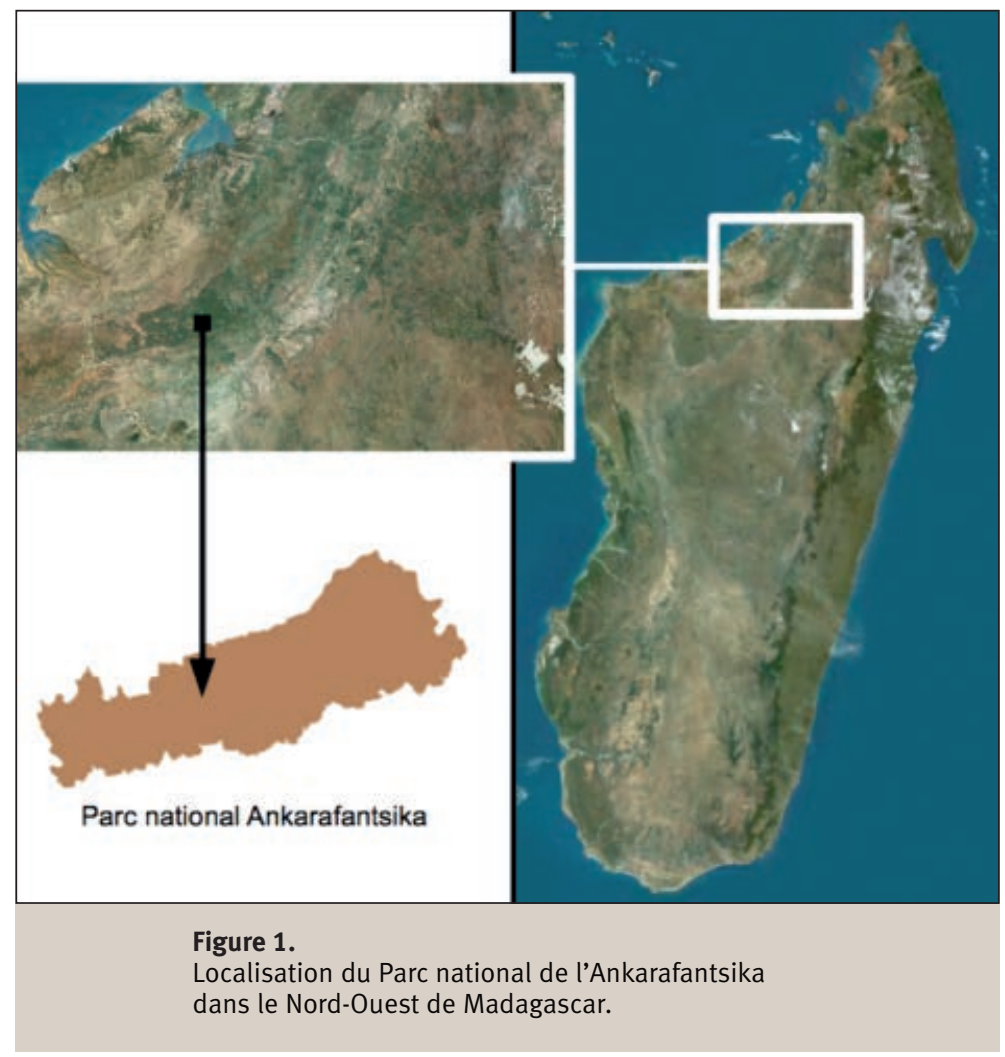

l'estimation des densités absolues n'est pas apparue indispensable. Dès lors, la méthode d'indice kilométrique d'abondance (Ika) a été utilisée, elle permet d'estimer efficacement l'abondance d'une espèce donnée dans un milieu (MATHOT et DOUCET, 2006). Cet indice est obtenu à partir du nombre d'observations directes ou indirectes (fèces, empreintes, fouilles, restes de repas) relevées sur des transects de longueur donnée :

lka total $=N / L$

où $N$ est le nombre total d'observations sur le transect et $L$ la longueur du transect en $\mathrm{km}$.

L'Ika consiste à accumuler un maximum de données en un temps réduit et à se baser uniquement sur les indices de présence animale sans les convertir en densité animale. Cette méthode supporte cependant un biais. En effet, le calcul de l'Ika néglige la diminution de la probabilité de détection des indices en fonction de la distance par rapport au transect. Cette probabilité de détection diminue d'autant plus vite que le milieu est fermé. Afin d'y pallier, il est envisageable de pondérer l'lka obtenu à l'aide d'un coefficient de visibilité. Cependant, GENET (2002) démontre que la visibilité en sous-bois n'influence pas significativement la valeur de l'Ika. Dès lors, en savane ouverte, l'Ika est un indicateur pertinent et stable pour estimer l'abondance des populations de potamochères et ses fluctuations dans le temps sans qu'il soit nécessaire de le pondérer avec un coefficient de visibilité.

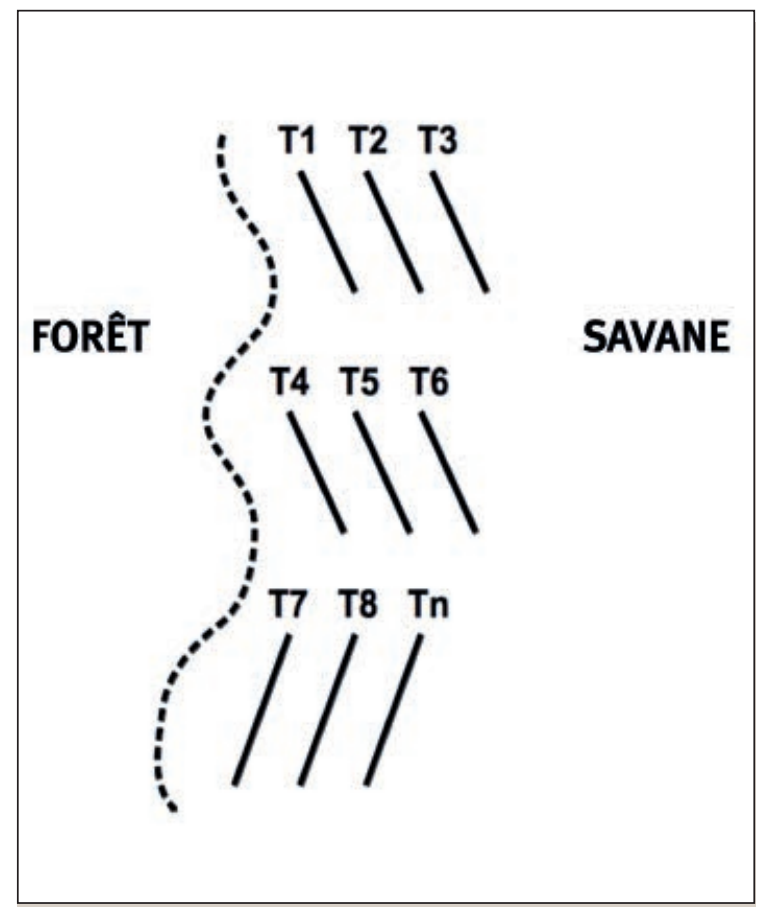

Figure 2.

Schéma de positionnement des transects en savane. 


\section{Collecte des données}

Au total, 18 transects de 300 m chacun ont été définis et six suivis par transect ont été réalisés à intervalles réguliers entre avril et août 2012, soit plus de 32 kilomètres parcourus. Le positionnement des transects s'est fait selon l'orientation prédominante de la zone (MARQUES et al., 2001). La forêt sèche étant le principal habitat structurant le site, les transects ont été disposés de façon parallèle à la lisière forestière du parc. Afin de prendre en compte l'effet de distance par rapport à cet habitat refuge pour les potamochères en journée, les transects ont été placés à intervalles réguliers depuis la lisière (200 m) (figure 2).

Depuis l'extrémité d'un transect, l'observateur principal suit la ligne de transect matérialisée par la succession de repères plastifiés disposés préalablement et ne s'en écarte jamais. L'observateur secondaire recherche les indices de présence des deux côtés de la ligne jusqu'à une distance approximative de $20 \mathrm{~m}$. Les deux observateurs marchent à allure réduite et constante pour maximiser la fiabilité de leurs relevés. Lorsqu'une trace de présence est détectée, celle-ci est rapportée sans distinction de nature. La collecte de données est répétée tous les 20 jours, intervalle de temps suffisant pour éviter une désaffection des sites par les potamochères du fait d'une présence accrue des observateurs.

Afin de permettre une analyse affinée des résultats, les 18 transects ont été catégorisés selon deux facteurs dominants du milieu : la présence de Strychnos spinosa (arbuste fruitier) et la présence de canyons érosifs (lavaka) (photos 3 et 4) sur les tracés des transects. Dans la savane ouverte étudiée, le milieu est particulièrement homogène ; peu de facteurs biotiques ou abiotiques sont susceptibles d'influencer l'abondance des potamochères. Seule la présence de nourriture (fruits de S. spinosa) et/ou de conditions topographiques particulières (canyons érosifs) permet une différenciation spatiale au sein de cette savane. Il est supposé ainsi que les fruits de $S$. spinosa pourraient conduire les suidés à fréquenter davantage les sites où ces arbustes sont présents, alors que la difficulté d'accès aux canyons érosifs devrait potentiellement y réduire leur présence.

\section{Analyse des données}

Les données du nombre de traces de potamochères ont été analysées en utilisant un modèle linéaire généralisé mixte. La variable réponse étant un comptage, il a été considéré que cette variable suit une distribution de Poisson et une fonction de lien « log » a été utilisée. Il a été tenu compte des problèmes de pseudo-réplication résultant de la répétition du protocole de comptage le long des mêmes transects à différentes dates en incluant un effet aléatoire transect dans le modèle. Enfin, pour obtenir des estimations pour un transect d'une longueur d'un kilomètre, alors que la longueur des transects utilisés dans l'étude était de 300 m, un terme d' " offset » égal à $\log (0,3)$ a été inclus dans le modèle. Les variables explicatives considérées étaient la variable binaire " canyon ", la variable binaire "spinosa » et enfin la variable quantitative « jour ». L'effet date n'est pas confondu avec l'effet S. spinosa car la variable « spinosa » reflète la présence de la plante mais pas la présence de ses fruits. Par conséquent, chaque transect est bien caractérisé par la présence de $S$. spinosa quelle que soit la période de l'année. Sur le plan biologique, l'effet date

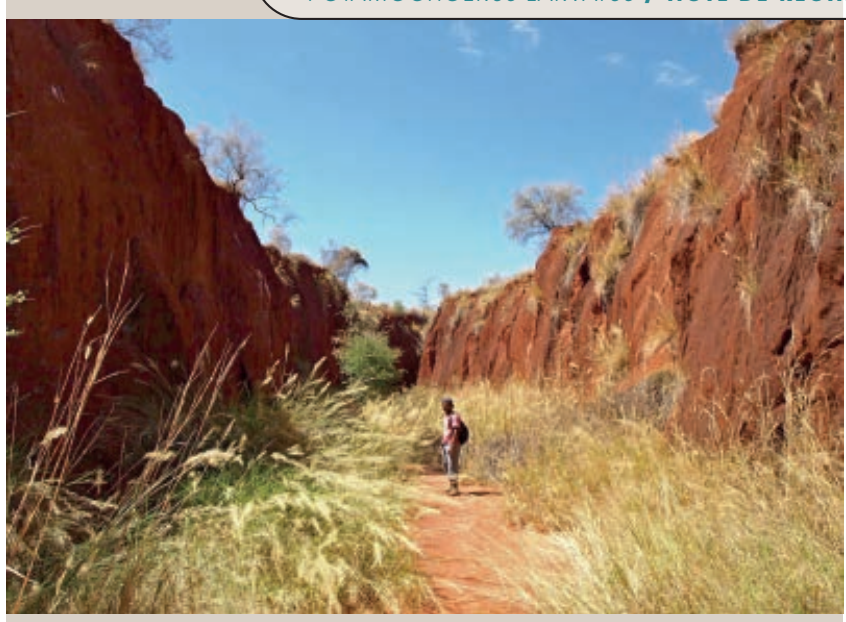

Photo 3.

Canyons érosifs (lavaka) en savane dans le Parc national de l’Ankarafantsika.

Photo A. Rouillé.

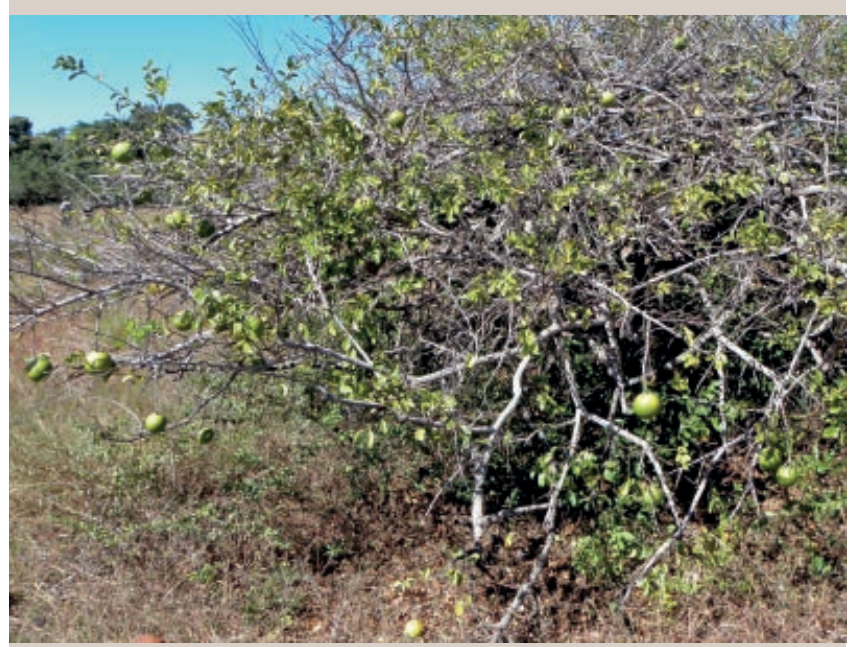

Photo 4.

Strychnos spinosa en période de fructification dans le Parc national de l'Ankarafantsika.

Photo M. Pedrono.

pourrait refléter la saisonnalité de la fructification. La valeur 1 a été attribuée à la variable « jour » pour le jour du premier comptage des traces de potamochères. Pour les itérations suivantes, la valeur de la variable « jour » correspond au nombre de jours écoulés depuis la date de ce premier comptage.

Le modèle statistique le plus complexe considéré dans l'analyse incluait les effets des trois variables explicatives mentionnées ci-dessus ainsi qu'une interaction entre la variable « jour » et la variable « spinosa ». Seule cette interaction a été considérée du fait de sa pertinence biologique. En effet, la fructification progressive de $S$. spinosa au cours de la période d'étude pourrait induire une attractivité croissante des transects contenant $S$. spinosa mais pas des autres transects. Étant donné la taille relativement faible de l'échantillon (18 transects), et dans un souci d'éviter la surparamétrisation du modèle statistique, seule cette interaction, biologiquement pertinente, a été testée. La significativité statistique de chacun des termes inclus dans le modèle décrit ci-dessus a été évaluée en utilisant le test du rapport de vraisemblance. Enfin, des estimations d'lka ont été dérivées du modèle ne contenant que les termes considérés comme statistiquement significatifs. 
Tableau I.

Significativité des variables explicatives des modèles.

\begin{tabular}{|c|c|c|c|}
\hline Variables & $\begin{array}{c}\text { Rapport de } \\
\text { vraisemblance }\end{array}$ & Ddl & p-valeur \\
\hline spinosa* & 8,53 & 1 & 0,003 \\
\hline canyon & 2,21 & 1 & 0,14 \\
\hline jour* & 9,57 & 1 & 0,002 \\
\hline spinosa : jour & & 1 & 0,09 \\
\hline
\end{tabular}

\section{Résultats}

Au total, 271 indices de présence de potamochères ont été comptabilisés durant la période d'étude. Aucun d'eux ne correspond à une observation visuelle directe. Les variables explicatives "spinosa » et " jour » se sont avérées avoir un effet significatif sur le nombre de traces détectées. L'effet de la variable " canyon " n'était pas significatif. L'interaction entre la variable « spinosa » et la variable « jour » était marginalement significative $(p=0,09)$ (tableau I).

Les estimations d'lka obtenues à partir du modèle n'incluant que les effets des variables "spinosa " et " jour» sans leur interaction sont présentées dans la figure 3. Les lignes en pointillé représentent l'intervalle de confiance à $95 \%$ de ces estimations ; le jour 1 correspond au 09/04/2012 et le jour 121 au 07/08/2012. L'Ika est supérieur le long des transects contenant des $S$. spinosa et augmente au cours de la période d'étude.

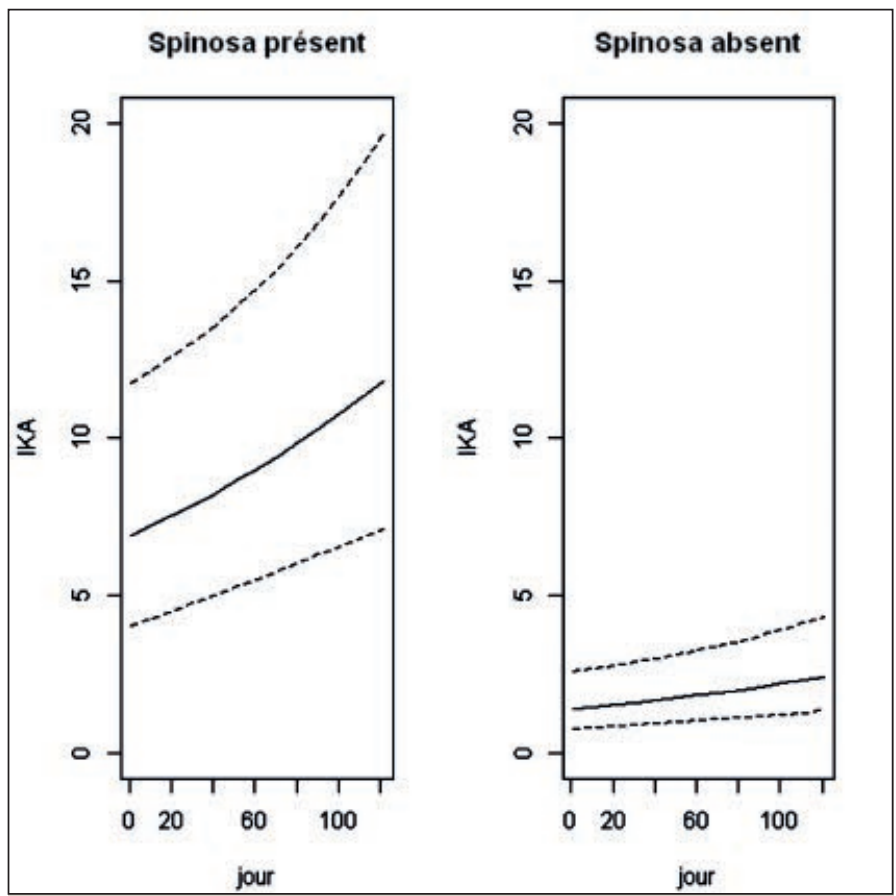

Figure 3.

Représentation graphique des estimations d'lka obtenues à partir du modèle incluant les variables « spinosa » et « jour » sans leur interaction.
La figure 4 représente les estimations d'lka dérivées du modèle contenant en plus l'interaction "spinosa : jour ». Selon ce modèle, la pente de la relation entre l'lka et la date est différente pour les transects avec et sans $S$. spinosa : l'lka augmente au cours de la période d'étude pour les transects avec $S$. spinosa alors qu'il est constant pour les transects sans $S$. spinosa. Bien que l'interaction «spinosa : jour » ne soit que marginalement significative, elle semble donc valider le lien étroit existant entre l'effet date et la phénologie de la fructification des S. spinosa (l'lka augmente en même temps que la saison de fructification progresse).

\section{Discussion et conclusion}

\section{Un indicateur biologique d'abondance}

L'augmentation significative d'abondance du potamochère observée durant la période d'étude révèle une présence accrue de cette espèce en savane à partir de la mimai. Cette augmentation n'est cependant observable que sur les sites caractérisés par la présence de Strychnos spinosa. L'augmentation d'abondance des potamochères notée durant cette période et les différences significatives d'abondance observées entre les transects démontrent que la fructification des Strychnos spinosa est le principal facteur influençant la variation d'abondance des populations de potamochères en savane dans notre étude. La présence de cet arbuste influence donc de façon importante la distribution spatiale et temporelle du potamochère dans le NordOuest de Madagascar.

De manière plus précise, l'lka est une méthode d'estimation de l'abondance prenant en compte un large panel d'indices de présence. Les observations directes en font partie. Or, durant toute la période de l'étude, aucune observation

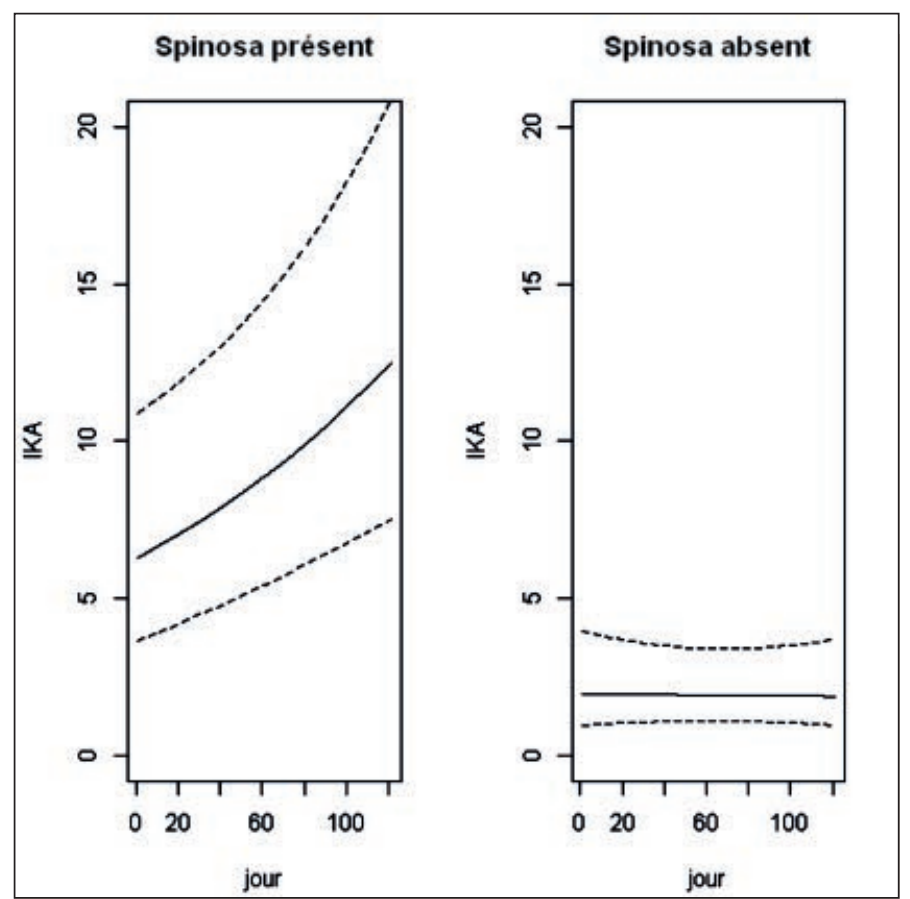

Figure 4.

Représentation graphique des estimations d'lka obtenues à partir du modèle incluant les variables « spinosa : jour » en interaction. 
de potamochères n'a été effectuée, ces animaux étaient absents des transects le jour lorsque les relevés étaient réalisés. Les savanes ne sont donc exploitées par les potamochères que durant leur phase d'activité nocturne et ils demeurent en forêt durant la journée.

L'augmentation d'abondance des populations de potamochères observée de la mi-mai à la mi-août renvoie à une attractivité alimentaire pour des fruits à maturité. Il existe d'autres espèces végétales fructifiantes dans les savanes de Madagascar, en particulier le jujubier commun Ziziphus jujuba (Rhamnaceae) qui, bien qu'exogène à Madagascar, pourrait potentiellement avoir un effet similaire sur l'augmentation d'abondance saisonnière des populations de potamochères.

\section{Recommandations en matière de gestion sanitaire}

La sympatrie ${ }^{2}$ entre porcs domestiques et suidés sauvages dans les savanes à Strychnos spinosa est susceptible de s'accroître durant leur fructification, et partant les risques de transmission de la Ppa, ou autres agents pathogènes, des porcs domestiques aux potamochères et inversement. La fructification de Strychnos spinosa peut ainsi être considérée comme un indicateur biologique d'abondance des populations de potamochères. II pourrait être utilisé pour cartographier les zones à risque (ex. densités de potamochères vs. populations de porcs domestiques et types d'élevage). Il peut également permettre d'adapter les pratiques d'élevage des porcins en limitant leur occupation des savanes à Strychnos spinosa lorsque leurs fruits sont à maturité afin de réduire les risques de transmission du virus de la Ppa. La coupe des arbres fruitiers sauvages en périphérie immédiate des villages permettrait également de réduire les risques de contacts entre suidés sauvages et domestiques.

La divagation des porcs domestiques reste cependant le facteur responsable d'un risque de partage du même espace. Bien que prohibée depuis l'arrêté interministériel 2082/00 du 8 mars 2000, l'errance des porcs est encore une pratique courante dans les élevages ruraux malgaches. La mise en œuvre d'une prophylaxie sanitaire relative à la Ppa doit, avec intérêt, prendre en compte cet aspect encore non résolu dans les villages.

S'ajouteront également aux pratiques d'élevage, les pratiques de chasse et de conservation des viandes (stockage vivant en enclos porcin) au titre des facteurs responsables d'un risque de transmission de pathogènes.

Le contrôle, voire l'éradication, de la Ppa en élevage porcin dépendra des modifications des pratiques d'élevage et de chasse à Madagascar (COSTARD et al., 2009b) comme dans le reste de l'Afrique (PENRITH et al., 2013). En ce sens, des campagnes de sensibilisation à l'épidémie de Ppa peuvent être envisagées en soutien aux mesures de régulation. La perception qu'ont les petits éleveurs de la situation enzootique est cruciale dans la lutte contre la Ppa. Une connaissance des enjeux communautaires et de l'incidence financière indirecte de la maladie sur les élevages (pertes de production) doit permettre d'influencer positivement les pratiques locales. La limitation des voies d'émission du virus de la Ppa entre réservoir potentiel sauvage et cheptel domestique est indispensable à la lutte contre l'épidémie.

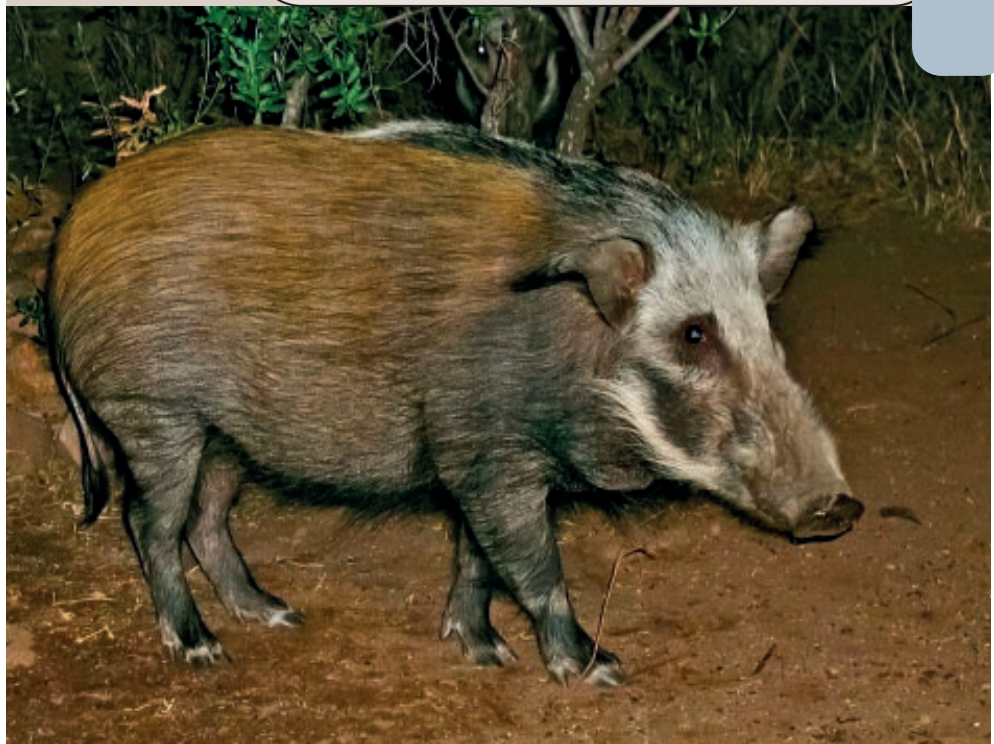

Photo 5.

Potamochère adulte, Potamochoerus larvatus.

Photo W. van Schalkwyk.

Cette étude écologique pourrait également servir de base à des études épidémiologiques d'autres maladies présentes à Madagascar comme la peste porcine classique (PATON, GREISER-WILKE, 2003) ou encore des infections et des infestations pour lesquelles il n'y a pas d'informations précises sur leurs occurrences chez le potamochère. Il s'agit en particulier de la cysticercose, zoonose parasitaire endémique majeure à Madagascar (MICHELET et al., 2010), de l'hépatite $E$, circulant dans les populations de porcs domestiques (TEMMAM et al., 2013), et de la trichinellose, dont la présence n'a pas été rapportée à Madagascar jusqu'à présent (DUPOUY-CAMET, 2000 ; POZIO, 2007), mais qui serait à rechercher chez les suidés domestiques et sauvages. Par ailleurs, la gale (Sarcoptic mange) est une dermatose parasitaire présente à Madagascar, en particulier chez les porcs domestiques (UILENBERG, 1969) ; transmissible à l'homme et décrite chez les lémuriens (LOUDON et al., 2006), elle n'a cependant jamais été documentée chez les potamochères. Une recherche sur cette infestation en tant que marqueur de contacts entre espèces (suidés sauvages/domestiques, suidés/hommes) trouverait également un grand intérêt dans la compréhension des voies de circulation de certaines maladies dans le pays. De tels travaux permettraient de tendre vers une approche holistique de la santé en adhérant à un modèle qui intégrerait la santé animale, la santé publique, et des approches écologiques.

\section{Remerciements}

Nous tenons à remercier particulièrement le Fofifa-Drzv et son directeur le Dr Rakotondravao, Madagascar National Parks et en particulier la Direction du Parc national de l'Ankarafantsika pour avoir permis cette étude, les habitants d'Ankazomborona pour leur aide, et V. Andrianjakarivelo (Wcs) pour les précieuses informations qu'il nous a fournies.

2 En biologie, deux espèces ou populations sont considérées comme sympatriques quand elles existent dans une même zone géographique et se rencontrent régulièrement ensemble. 


\section{Références bibliographiques}

ALONSO C., 2013. African swine fever virus conquers new territories. Virus Research, 173 (1): 1-2.

AMILLS M., RAMIREZ O., GALMAN-OMITOGUN O., CLOP A., 2013. Domestic pigs in Africa. African Archaeological Review, 30 (1): 73-82. ANDRIANJAKARIVELO V., 1999. L'importance socio-économique de la chasse aux sangliers et son impact sur la densité des sangliers à Ambanizana et Sahamalaza (Péninsule de Masoala) durant l'année 1998. Rapport non publié pour la Wildlife Conservation Society, Antananarivo, Madagascar, $14 \mathrm{p}$.

ANDRIANJAKARIVELO V., 2000. Rôle écologique des potamochères dans la Réserve Spéciale de Nosy Mangabe. Rapport non publié pour la Wildlife Conservation Society, Antananarivo, Madagascar, 31 p.

ANDRIANJAKARIVELO V., 2003. Artiodactyla: Potamochoerus larvatus, bush pig. In: Goodman S. M., Benstead J. P. (eds). The natural history of Madagascar. Chicago, États-Unis, The University of Chicago Press, 1365-1367.

ANGAP, 2007. Plan de gestion de la conservation, Parc National Ankarafantsika. Antananarivo, Madagascar, Association nationale pour la gestion des aires protégées (Angap), $94 \mathrm{p}$.

BURNEY D. A., BURNEY L. P., GODFREY L. R., JUNGERS W. L., GOODMAN S. M., WRIGHT H. T., JULL A. J. T., 2004. A chronology for late prehistoric Madagascar. Journal of Human Evolution, 47 (1-2): 25-63.

COSTARD S., PORPHYRE V., MESSAD S., RAKOTONDRAHANTA S., VIDON H., ROGER F., PFEIFFER D. U., 2009a. Multivariate analysis of management and biosecurity practices in smallholder pig farms in Madagascar. Preventive Veterinary Medicine, 92 (3): 199-209.

COSTARD S., WIELAND B., DE GLANVILLE W., JORI F., ROWLANDS R. J., VOSLOO W., ROGER F., PFEIFFER D. U., DIXON L. K., 2009b. African swine fever: how can global spread be prevented? Philosophical Transactions of the Royal Society of London B, 364, 2683-2696. COSTARD S., MUR L., LUBROTH J., SANCHEZ-VIZCAINO J. M., PFEIFFER D. U., 2013. Epidemiology of African swine fever virus. Virus Research, 173 (1): 191-197.

DUPOUY-CAMET J., 2000. Trichinellosis: a worldwide zoonosis. Veterinary Parasitology, 93 (3-4): 191-200.

GARBUTT N., 2007. Mammals of Madagascar: a complete guide. Yale, États-Unis, Yale University Press, 304 p.

GARCIA G., GOODMAN S. M., 2003. Hunting of protected animals in the Parc National d'Ankarafantsika, north-western Madagascar. Oryx, 37 (1): 115-118.

GENET H., 2002. Gestion de la faune dans les concessions forestières du Gabon. Mémoire de fin d'études, Faculté universitaire des sciences agronomiques de Gembloux, Belgique, $78 \mathrm{p}$.

GONZAGUE M., ROGER F., BASTOS A., BURGER C., RANDRIAMPARANY T., SMONDACK S., CRUCIĖRE C., 2001. Isolation of a non-haemadsorbing, non-cytopathic strain of African swine fever virus in Madagascar. Epidemiology and Infection, 126 (3): 453-459. HUNTER A., ULINBERG G., MEYER C., BOURDOISEAU G., BOUYER J., CHANTAL J. H., DORCHIES P., FAYE B., RICHARD D., ROGER F., STACHURSKI F., TILLARD E., 2006. La santé animale. Vol. 2. : Principales maladies. Versailles, France, Éditions Quæ, coll. Agricultures tropicales en poche, $312 \mathrm{p}$.

JORI F., BASTOS A. D. S., 2009. Role of wild suids in the epidemiology of African swine fever. EcoHealth, 6 (2): 296-310.

JORI F., VIAL L., PENRITH M. L., PEREZ-SANCHEZ R., ETTER E., ALBINA E., MICHAUD V., ROGER F., 2013. Review of the sylvatic cycle of African swine fever in sub-Saharan Africa and the Indian Ocean. Virus Research, 173 (1): 212-227.

LOUDON J. E., SAUTHER M. L., FISH K. D., HUNTER-ISHIKAWA M., 2006. One reserve, three primates: applying a holistic approach to understand the interconnections among ring-tailed lemurs (Lemur catta), Verreaux's sifaka (Propithecus verreauxi), and humans (Homo sapiens) at Beza Mahafaly Special Reserve, Madagascar. Ecological and Environmental Anthropology, 2 (2): 53-74.
MARQUES F. C., BUCKLAND S. T., GOFFIN D., DIXON C. A., BORCHERS D. R., MAYLE B. A., PEACE A. J., 2001. Estimating deer abundance from line transect surveys of dung: sika deer in southern Scotland. Journal of Applied Ecology, 38 (2): 349-363.

MATHOT L., DOUCET J.-L., 2006. Méthode d'inventaire faunique pour le zonage des concessions en forêt tropicale. Bois et Forêts des Tropiques, 287 (1) : 59-70.

MENG X. J., LINDSAY D. S., SRIRANGANATHAN N., 2009. Wild boars as sources for infectious diseases in livestock and humans. Philosophical Transactions of the Royal Society of London B, 364 (1530): 2697-2707.

MICHELET L., CAROD J.-F., RAKONTONDRAZAKA M., MA L., GAY F., DAUGA C., 2010. The pig tapeworm Taenia solium, the cause of cysticercosis: Biogeographic (temporal and spacial) origins in Madagascar. Molecular Phylogenetics and Evolution, 55 (2): 744-750. PATON D., GREISER-WILKE I., 2003. Classical swine fever - an update. Research in Veterinary Science, 75 (3): 169-178.

PENRITH M.-L., VOSLOO W., JORI F., BASTOS A. D. S., 2013. African swine fever virus eradication in Africa. Virus Research, 173 (1): 228-246.

PENRITH M. L., THOMSON G. R., BASTOS A. D. S., 2004. African swine fever. In: Coetzer J. A., Tustin R. C. (eds). Infectious diseases of livestock with special reference to Southern Africa. Cape Town, Afrique du Sud, Oxford University Press, 1088-1119.

POZIO E., 2007. World distribution of Trichinella spp. infections in animals and humans. Veterinary Parasitology, 149: 3-21.

RAVAOMANANA J., JORI F., VIAL L., PÉREZ-SANCHEZ R., BLANCO E., MICHAUD V., ROGER F., 2011. Assessment of interactions between African swine fever virus, bushpigs (Potamochoerus larvatus), Ornithodoros ticks and domestic pigs in north-western Madagascar. Transboundary and Emerging Diseases, 58 (3): 247-254.

RAVAOMANANA J., MICHAUD V., JORI F., ANDRIATSIMAHAVANDY A., ROGER F., ALBINA E., VIAL L., 2010. First detection of African Swine Fever Virus in Ornithodoros porcinus in Madagascar and new insights into tick distribution and taxonomy. Parasites and Vectors, 3: 115. ROGER F., RATOVONJATO J., VOLA P., UILENBERG G., 2001. Ornithodoros porcinus ticks, bushpigs, and African swine fever in Madagascar. Experimental and Applied Acarology, 25: 263-269.

ROGER F., CRUCIĖRE C., RANDRIAMAHEFALA N., ZELLER H., UILENBERG G., RANDRIAMPARANY T., GONZAGUE M., ROUSSET D., BENKIRANE A., DIALLO A., 2000. African swine fever in Madagascar: epidemiological assessment of the recent epizootic. In: Proceedings of the 9th International Symposium on Veterinary Epidemiology and Economics, Breckenridge, Colorado, USA. www.sciquest.org.nz. ROUSSET D., RANDRIAMPARANY T., MAHARAVO RAHANTAMALALA C. Y., RANDRIAMAHEFA N., ZELLER H., RAKOTO ANDRIANARIVELO M., ROGER F., 2001. Introduction de la peste porcine africaine à Madagascar, histoire et leçons d'une émergence. Archives de l'Institut Pasteur de Madagascar, 67 (1-2) : 31-33.

SEYDACK A. H. W., 1990. Ecology of the bushpig Potamochoerus porcus Linn. 1758 in the Cape Province, South Africa. Thèse, université de Stellenbosch, Afrique du Sud, 728 p.

TEMMAM S., BESNARD L., HÉRAUD J. M., ANDRIAMANIVO H. R., FORAY C., ANDRIAMANDIMBY S. F., RAFISANDRANTANTSOA J. T., CARDINALE E., DELLAGI K., PAVIO N., PASCALIS H., PORPHYRE V., 2013. High prevalence of Hepatitis $E$ in humans and pigs and evidence of genotype-3 virus in swine, Madagascar. The American Journal of Tropical Medicine and Hygiene, 88 (2): 329-338.

UILENBERG G., 1969. Inventaire des arthropodes, protozoaires et rickettsiales parasites des animaux domestiques et des animaux de laboratoire à Madagascar. Archives de l'Institut Pasteur de Madagascar, 38 (1) : 69-105. 